\title{
Boron Nitride Nanotube Impurity Detection and Purity Verification
}

Mahmoud S. Amin, ${ }^{1}$ Tucker Ethan Molin, ${ }^{2}$ Carlo Tampubolon, ${ }^{2}$ David E. Kranbuehl, ${ }^{2, *}$ Hannes C. Schniepp ${ }^{1}$

1) Department of Applied Science, The College of William \& Mary

2) Department of Chemistry, The College of William \& Mary

*) dekran@wm.edu

\section{Supplementary Information}

S1: Boron Nitride Impurity removal verification

As detection of boron nitride impurities' presence and removal, is primarily accomplished with Raman Spectroscopy, a method is needed to establish what percentage of the boron nitride impurities can be verified to have been removed by the heptane purification method through Raman Spectroscopy. To this end, we have chosen the Raman peaks of each BNNT material and run an analysis whereby the noise level of a given spectrum was measured and then compared to the $1365 \mathrm{~cm}^{-1}$ peak height of the boron nitride impurity. This gives us a measure of what percentage of the boron nitride impurities can be said to have been removed.

Each peak was fitted with a Lorentzian fit and then the displayed adjoining region was used to acquire the noise level. The root mean square of the noise level residuals after subtracting the peak fit was then divided by the peak height of the boron nitride impurity peak to find the noise to peak height ratio, Subtracting that ratio from 1 yields an estimate of the percent of boron nitride impurities that has been removed. Clearly the smaller the boron nitride impurity peak the greater the relative noise level and the resulting estimate of boron nitride impurity removed by the purification process is decreased.

\begin{tabular}{|l|l|l|l|}
\hline & $\begin{array}{l}\text { Noise level (Root } \\
\text { Mean Square) }\end{array}$ & $\begin{array}{l}1365 \mathrm{~cm}^{-1} \text { Peak } \\
\text { Height }\end{array}$ & $\begin{array}{l}\text { Percent Verified } \\
\text { Removed }\end{array}$ \\
\hline BNNT 1 & 62.39 & 3974.77 & $98.43 \%$ \\
\hline BNNT 2 & 194.72 & 971.59 & $79.96 \%$ \\
\hline BNNT 3 & 97.25 & 1880.86 & $94.83 \%$ \\
\hline
\end{tabular}


(a)

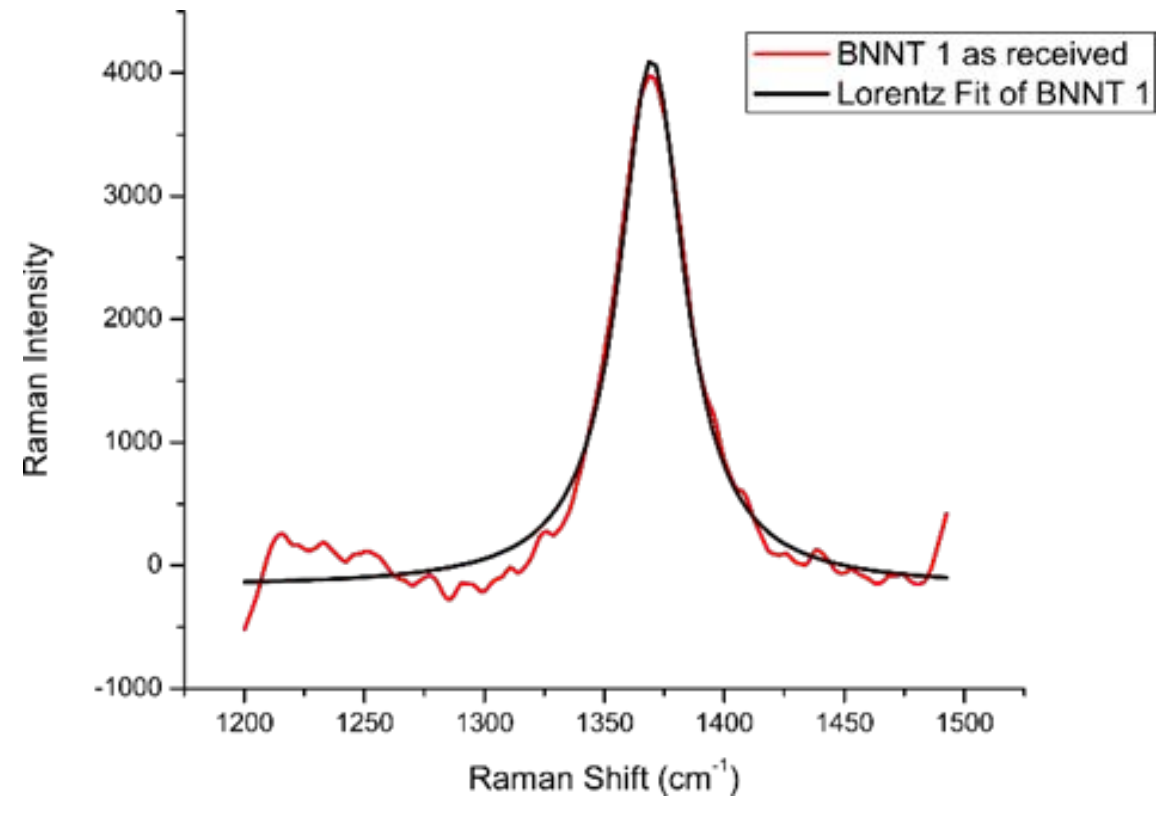

(b)

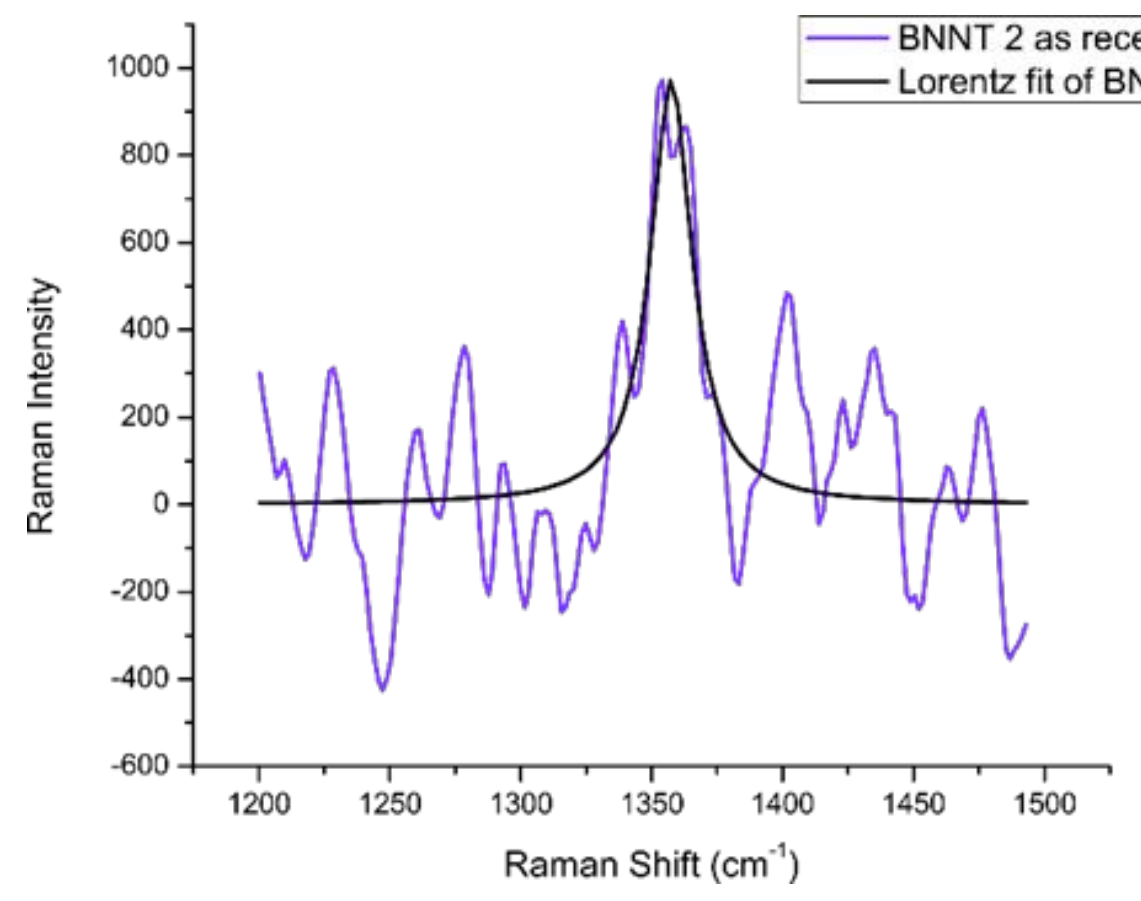

Figure S1: (a) Raman spectrum of BNNT 1 as-received, centered around the boron nitride impurity peak with the Lorentzian fit of the spectrum. (b) Raman spectrum of BNNT 2 as-received, centered around the boron nitride impurity peak with the Lorentzian fit of the spectrum. 
(c)

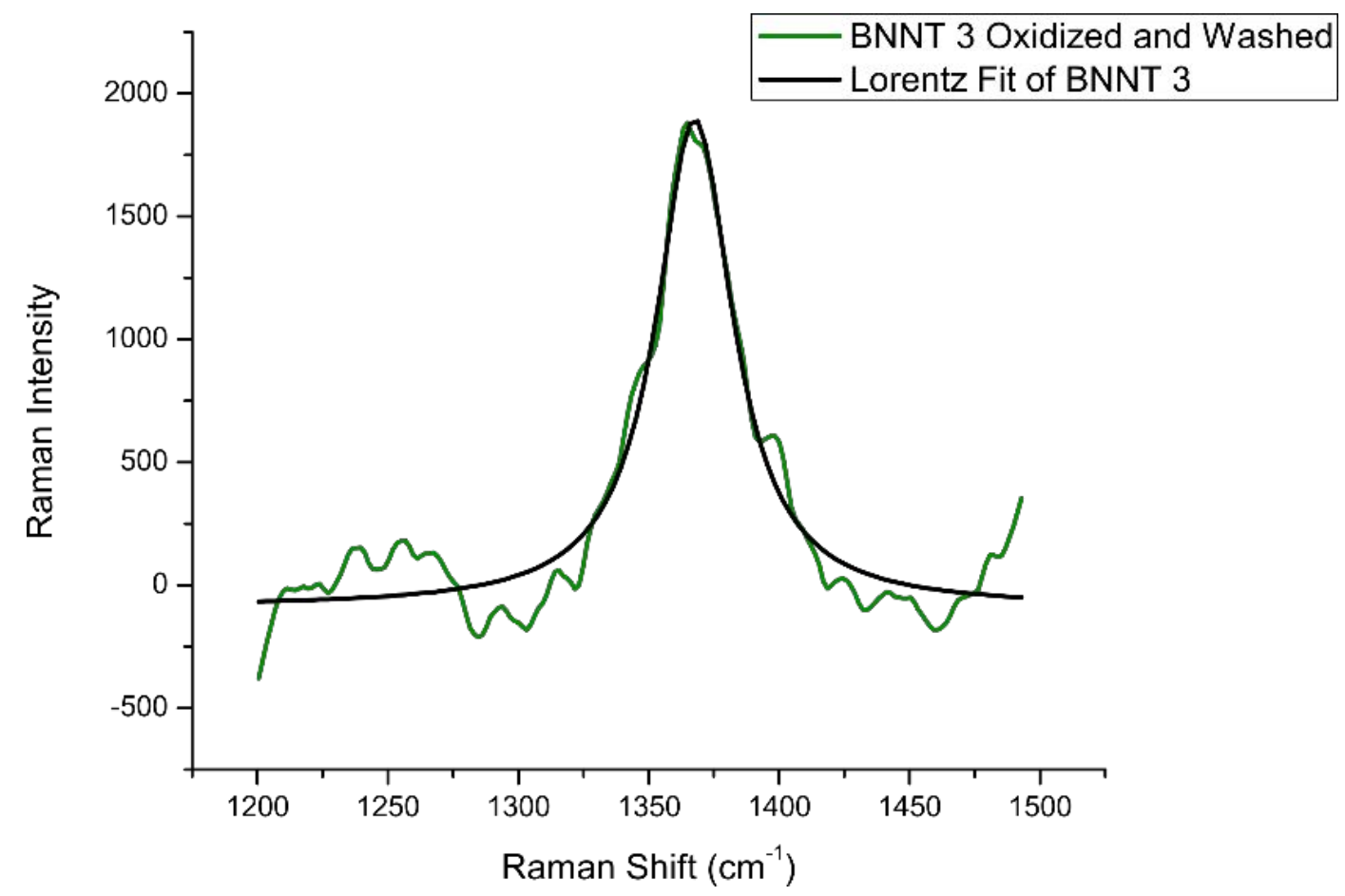

Figure S1: (c) Raman spectrum of BNNT 3 Oxidized and Washed, centered around the boron nitride impurity peak with the Lorentzian fit of the spectrum. 


\section{S2: Boron Oxide Removal Verification}

As discussed within the paper, the primary use of FTIR was to verify the presence and removal of boron oxide and to quantify the degree of removal based on FTIR measurements. As such, analysis was performed to quantify the degree of removal based on FTIR measurements. The samples analyzed were BNNT 1 as produced and BNNT 3 as produced. This analysis was done on the FTIR absorbance spectra after all of the $1300 \mathrm{~cm}^{-1}$ peaks were normalized to be approximately the same height. A Lorentzian fit was performed on the Boron Oxide peak $\left(3200 \mathrm{~cm}^{-1}\right)$ and then subtracted from the measured spectra to produce residual plots. A measurement of the noise level of the $3200 \mathrm{~cm}^{-1}$ peak was taken from an area of the residual plot which features no peaks but is close to the $3200 \mathrm{~cm}^{-1}$ peak using the root mean square of absorbance values within that area. The ratio of the noise level to the peak height was then used to calculate what percentage of the boron oxide that was removed.

\begin{tabular}{|l|l|l|}
\hline Sample Name & BNNT 1 As Produced & BNNT 3 As Produced \\
\hline Peak Height & 0.035 & 0.11 \\
\hline Noise Sampling Range & $2800-2900\left(\mathrm{~cm}^{-1}\right)$ & $3550-3700\left(\mathrm{~cm}^{-1}\right)$ \\
\hline Noise level (Root mean square) & 0.00058 & 0.00072 \\
\hline Percent Verified Removed & $98.37 \%$ & $99.35 \%$ \\
\hline
\end{tabular}


(a)

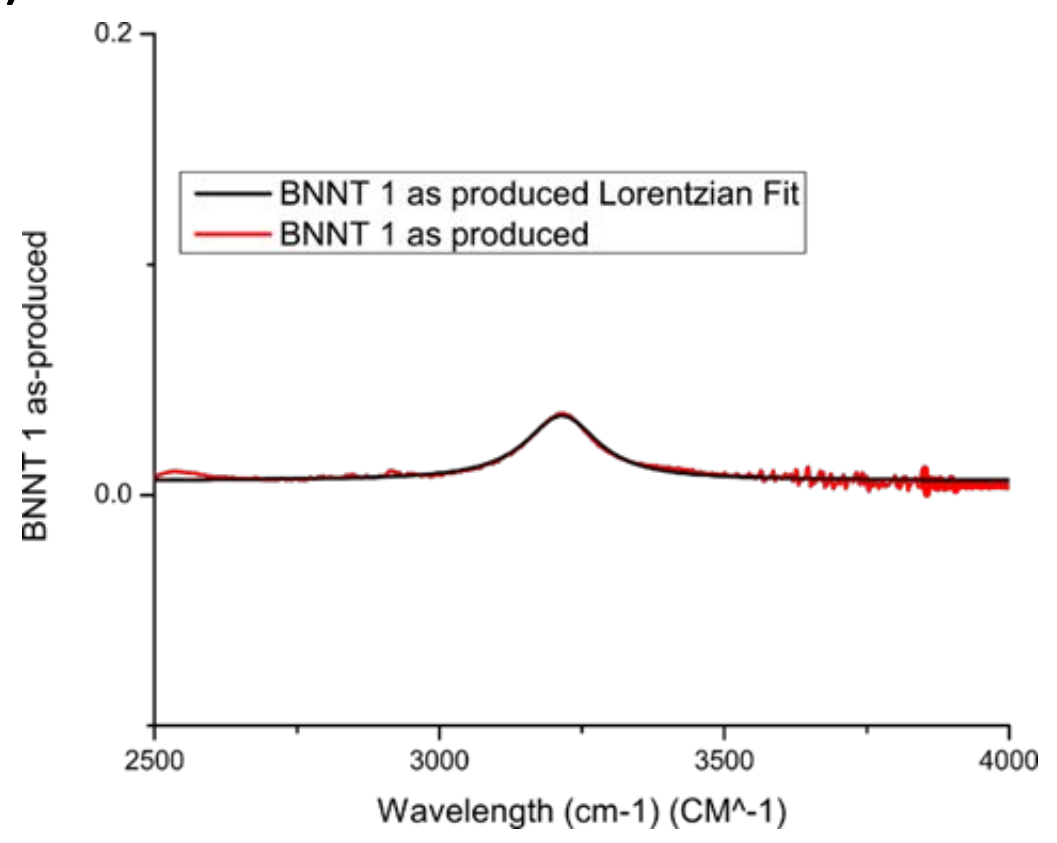

(b)

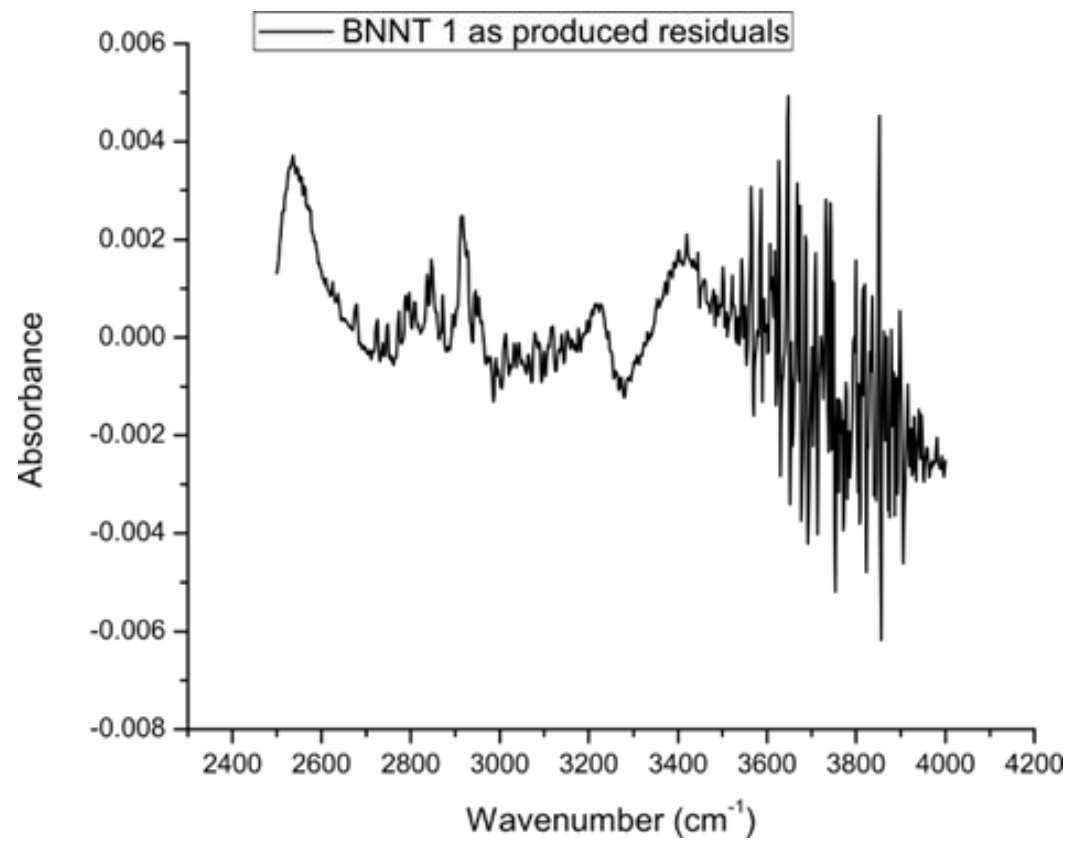

Figure S2: (a) FTIR Spectrum of BNNT 1, range $2500-4000 \mathrm{~cm}^{-1}$ (red) with a Lorentzian fit (black). (b) residual plot of the Lorentzian fit of the $\mathrm{B} 2 \mathrm{O} 3$ peak 
(a)

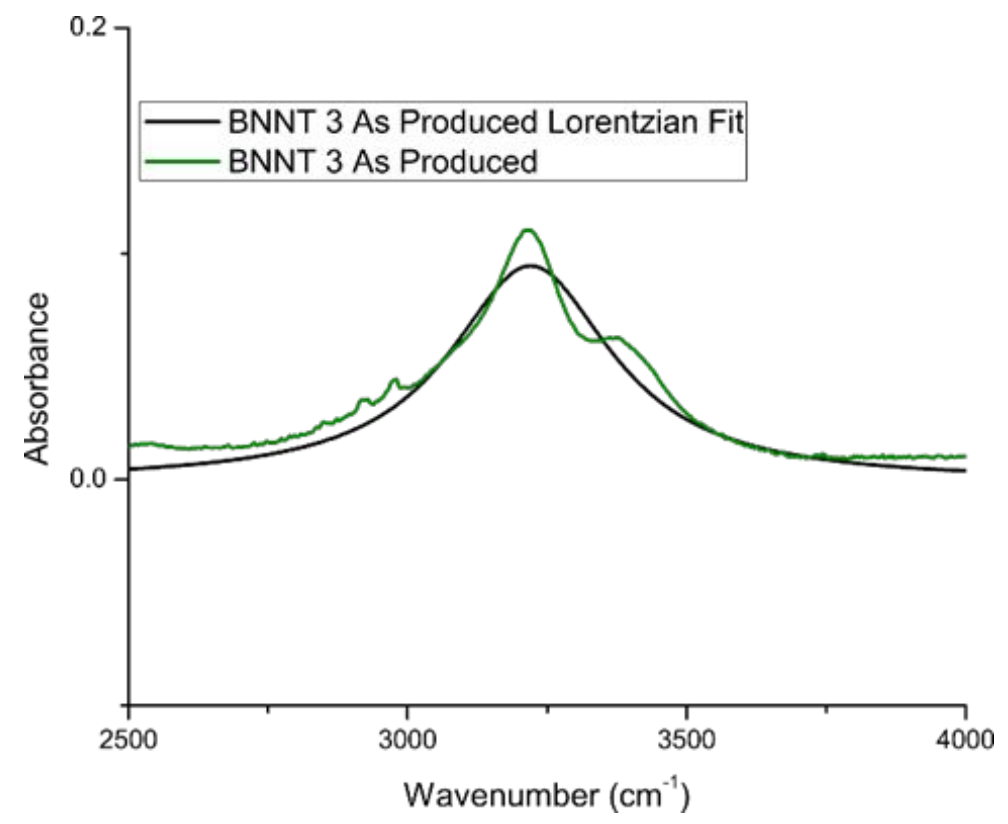

(b)

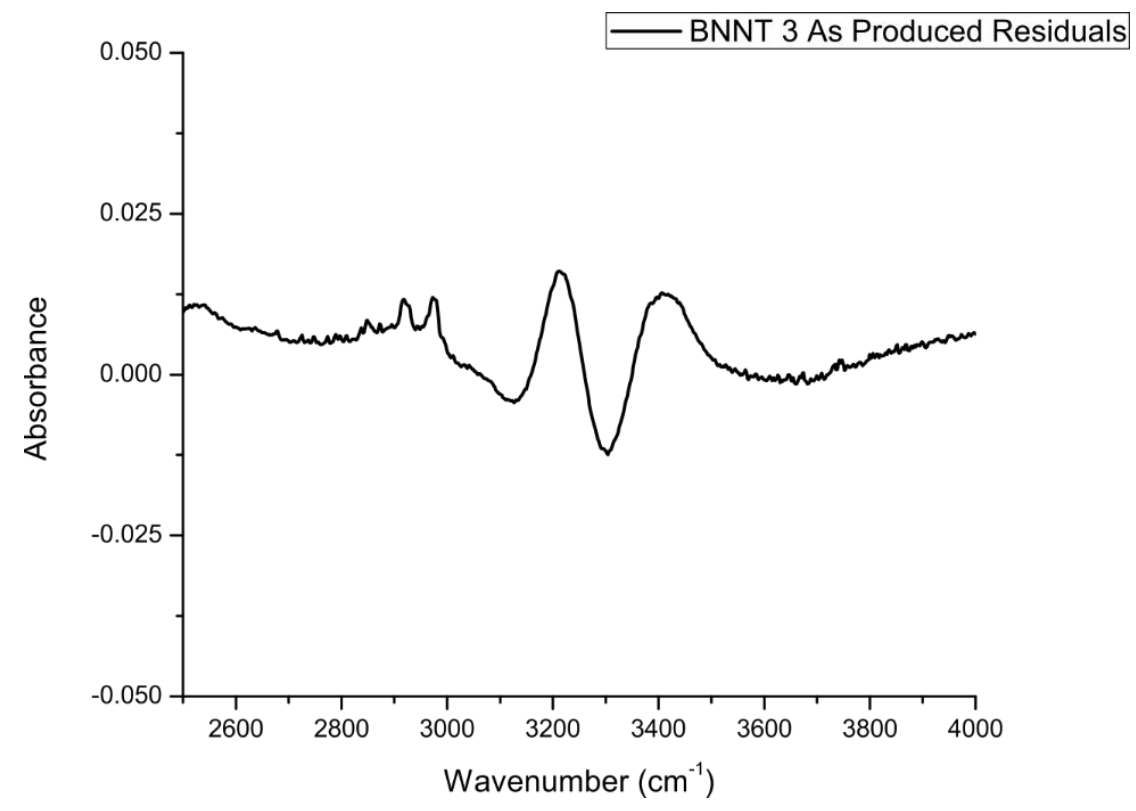

Figure S2: (c) FTIR Spectrum of BNNT 3, range $2500-4000 \mathrm{~cm}^{-1}$ (green) with a Lorentzian fit (black). (b) residual plot of the Lorentzian fit of the $\mathrm{B} 2 \mathrm{O} 3$ peak 
Table S3: Table of normalized FTIR absorbance peak heights and areas and their ratios

\begin{tabular}{|l|l|l|l|l|l|l|l|}
\hline $\begin{array}{l}\text { Sample } \\
\text { Name }\end{array}$ & $\begin{array}{l}\text { BNNT 1 } \\
\text { as received }\end{array}$ & $\begin{array}{l}\text { BNNT 1 } \\
\text { heptane } \\
\text { purified }\end{array}$ & $\begin{array}{l}\text { BNNT 2 } \\
\text { as received }\end{array}$ & $\begin{array}{l}\text { BNNT 2 } \\
\text { Heptane } \\
\text { Purified }\end{array}$ & $\begin{array}{l}\text { BNNT 3 } \\
\text { Oxidized and } \\
\text { washed }\end{array}$ & $\begin{array}{l}\text { BNNT 3 } \\
\text { Heptane } \\
\text { Purified }\end{array}$ & h-BN \\
\hline $\begin{array}{l}780 \text { Peak } \\
\text { Height }\end{array}$ & 0.50 & 0.56 & 0.45 & 0.75 & 0.52 & 0.67 & 1.10 \\
\hline $\begin{array}{l}1300 \text { Peak } \\
\text { Height }\end{array}$ & 1.01 & 1.01 & 1.01 & 1.01 & 1.01 & 1.01 & 1.01 \\
\hline $\begin{array}{l}780 / 1300 \\
\text { Peak Height } \\
\text { Ratio }\end{array}$ & 0.50 & 0.55 & 0.45 & 0.74 & 0.51 & 0.67 & 1.08 \\
\hline $\begin{array}{l}780 \text { Peak } \\
\text { Area }\end{array}$ & 95.06 & 46.34 & 61.94 & 67.87 & 70.60 & 52.78 & 181.29 \\
\hline $\begin{array}{l}1300 \text { Peak } \\
\text { Area }\end{array}$ & 242.81 & 236.30 & 209.37 & 261.50 & 241.05 & 240.67 & 452.90 \\
\hline $\begin{array}{l}780 / 1300 \\
\text { Peak Area } \\
\text { Ratio }\end{array}$ & 0.39 & 0.20 & 0.30 & 0.26 & 0.29 & 0.22 & 0.40 \\
\hline
\end{tabular}

S3: The FTIR Spectra of the three BNNT batches were taken and their spectra normalized such that the $1300 \mathrm{~cm}^{-1}$ peak of each spectra was equal. Their $780 \mathrm{~cm}^{-1}$ peak height was then measured and the ratio of the $780 \mathrm{~cm}^{-1}$ peak to the $1300 \mathrm{~cm}^{-1}$ peak was then taken. These peaks were then given a multiple peak Lorentzian fit and their areas compared in the same manner as the heights.

The ratio of the heights and the ratio of the peak areas for the $1300 \mathrm{~cm}^{-1}$ and the $780 \mathrm{~cm}^{-1}$ peak were determined. As the table shows the ratios of the peak heights did not show a consistent decreasing trend as the boron nitride impurities were removed following heptane purification. On the other hand, the peak area ratio do show a decreasing change. Thus for these BNNT materials a decreasing area ratio was an indication of boron nitride impurity removal 